\title{
Magnetization Reversal in Hexagonal Nanomagnets
}

\author{
N. DAng XuAn ${ }^{a}$, Ch. DÖPKE ${ }^{a}$, T. Blachowicz ${ }^{b}$ And A. Ehrmann ${ }^{a} * *$ \\ ${ }^{a}$ Bielefeld University of Applied Sciences, Faculty of Engineering and Mathematics, 33619 Bielefeld, Germany \\ ${ }^{b}$ Silesian University of Technology, Institute of Physics - Center for Science and Education, 44-100 Gliwice, Poland
}

(Received October 28, 2019; revised version November 25, 2019; in final form November 27, 2019)

Magnetic nanostructures often exhibit interesting shape anisotropies, which may provide new possible applications, depending on the geometry and the magnetic material with its magnetocrystalline anisotropy. Comparing the pure magnetic materials iron, cobalt, and nickel, their anisotropy constants vary by approximately two orders of magnitude, allowing for testing the effect of superposing shape anisotropies with different magnetocrystalline anisotropies. Here we report on angle-dependent micromagnetic simulations of three different hexagonal-shaped nanomagnets, prepared from iron, cobalt, and nickel. While usual hysteresis loops, mostly without steps, are visible for nickel nanomagnets, cobalt results in a broad range of magnetization reversal processes with several steps which vary during repeated simulations due to variations of the anisotropy axes in different grains of the nanoparticles. Iron provides the best compromise between steps along the hysteresis loops which were proven to be correlated with stable intermediate states, usable for quaternary or higher-order storage devices, and reliable magnetization reversal processes even for sputtered samples with arbitrary anisotropy orientations in the single grains. Our examinations reveal that for nanomagnets on dimensions of a few hundred nanometers, iron is the ideal material not only for new magnetic data storage applications, but also for basic investigations of new and possibly technologically usable magnetization reversal processes.

DOI: 10.12693/APhysPolA.137.395

PACS/topics: magnetic anisotropy, magnetic domains, domain walls, ferromagnetism, micromagnetism

\section{Introduction}

Magnetic nanostructures are of high interest for basic research of the interplay between magnetocrystalline and shape anisotropy [1] as well as for a broad range of applications from biomedicine to data storage systems [2]. Recently, often magnetic nanofibers were investigated, e.g. fibers with structured "nanotraps" which enabled multiple magnetic states [3]. On the other hand, bent nanofibers offer interesting magnetization reversal processes $[4,5]$ and domain wall propagation modes $[6,7]$.

While single magnetic nanowires are often correlated with the racetrack memory, using domain wall propagation for data storage and manipulation [8-10], the combination of two or more magnetic nanowires allows for creating quaternary and higher-order memory devices. This idea is similar to the intermediate resistance states recently found in $\mathrm{Cu} /$ cobalt ferrite/Pt sandwich structure which could also be used for multilevel resistive switching [11].

Such open frames in square [12] or hexagonal shape [13] from iron $(\mathrm{Fe})$ were shown to exhibit one or more steps along the slope of the hysteresis loop, often correlated with stable intermediate states which can be used as additional data storage state, enabling storing two or more bits in one storage position. For other materials, such as nickel, stable intermediate states do not necessarily occur [14].

\footnotetext{
* corresponding author; e-mail: andrea.ehrmann@fh-bielefeld.de
}

Closed square Fe nanodots did not reveal such stable intermediate states, either [15]. However, since closed areas are easier to produce lithographically than frames with thin "walls", such nanoparticles would be of interest for bit-patterned media and other applications if they also showed more than the common two magnetic states at vanishing external magnetic field. In addition, other magnetic materials may offer new magnetization reversal processes, such as triangular permalloy microparticles which showed magnetization reversal either stepwise or via an intermediate state, depending on the particle orientation [16]. Cobalt, on the other hand, was found to exhibit different intermediate states for diverse shapes $[17,18]$.

Here we report on micromagnetic simulations of nanoparticles of three hexagonal shapes, modelled with nickel $(\mathrm{Ni})$, iron and cobalt $(\mathrm{Co})$ to span a broad range of magnetocrystalline anisotropies, investigated in dependence of the in-plane angle of the external magnetic field. In this way, an overview can be given under which conditions the interplay between shape and material result in stable intermediate states which may be used for new storage devices.

\section{Simulations}

For the micromagnetic simulations described here, the micromagnetic simulation program Object Oriented MicroMagnetic Framework (OOMMF) was used [19]. For the materials under examination, the original OOMMF material parameters were adopted, corresponding to typical literature values [20-22]: 
- Ni: $M_{s}=490 \times 10^{3} \mathrm{~A} / \mathrm{m}, A=9 \times 10^{-12} \mathrm{~J} / \mathrm{m}$, $K_{1}=-5.7 \times 10^{3} \mathrm{~J} / \mathrm{m}^{3}$,

- Fe: $M_{s}=1700 \times 10^{3} \mathrm{~A} / \mathrm{m}, A=21 \times 10^{-12} \mathrm{~J} / \mathrm{m}$, $K_{1}=48 \times 10^{3} \mathrm{~J} / \mathrm{m}^{3}$,

- Co: $M_{s}=1400 \times 10^{3} \mathrm{~A} / \mathrm{m}, A=30 \times 10^{-12} \mathrm{~J} / \mathrm{m}$, $K_{1}=520 \times 10^{3} \mathrm{~J} / \mathrm{m}^{3}$,

where $M_{s}$ is the magnetization at saturation, $A$ is the exchange constant, and $K_{1}$ is the magnetocrystalline anisotropy constant. It should be mentioned that $\mathrm{Ni}$ and Fe have a cubic anisotropy while Co shows a uniaxial anisotropy.

The fundamental equation for simulation is the Landau-Lifshitz-Gilbert (LLG) consisting of the precessional and damping terms set at the temperature $T=0 \mathrm{~K}$. The measure of the damping is represented by the phenomenological Gilbert constant. It should be mentioned that while the saturation magnetization $M_{s}$ and the exchange constant $A$ are of the same order of magnitude for all three materials, the anisotropy constant $K_{1}$ is growing by approximately one order of magnitude from $\mathrm{Ni}$ to $\mathrm{Fe}$ and from $\mathrm{Fe}$ to $\mathrm{Co}$, suggesting a significantly reduced influence of the magnetocrystalline anisotropy in Ni nanoparticles, as compared to Fe, while the magnetocrystalline anisotropy should be clearly dominating in the Co nanoparticles under investigation. The Gilbert damping constant $\alpha$ was set to 0.5 (quasistatic case), and the mesh size of the elementary cube was equal to $d=5 \mathrm{~nm}$. To model sputtered systems without thermal after-treatment, random anisotropy axes were chosen, in the meaning of random distribution of the axis orientations between cubes creating the meshed samples. Maximum simulated magnetic fields were chosen between $100 \mathrm{mT}$ and $1 \mathrm{~T}$, depending on the material, always ensuring that the saturation was reached, i.e. that magnetization reversal was completed.

Sample orientations were varied between $0^{\circ}$ and $90^{\circ}$. As a result, longitudinal magnetization components $M_{L}$ and transverse magnetization components $M_{T}$ are given, referenced to the external magnetic field direction, as usual, with $M_{L}$ being parallel to the external magnetic field and $M_{T}$ being perpendicular to it.

The three different shapes under examination are depicted in Fig. 1a-c. The thickness was always chosen as $5 \mathrm{~nm}$. To make the three shapes comparable, their areas were calculated to be identical, resulting in the following lateral dimensions of the rectangle covering the figures:

- equilateral: width $500 \mathrm{~nm}$, height $500 \mathrm{~nm}$

- rectangular: width $360 \mathrm{~nm}$, height $785 \mathrm{~nm}$

- concave: width $610 \mathrm{~nm}$, height $530 \mathrm{~nm}$

\section{Results and discussion}

Firstly, Fig. 2 depicts exemplary hysteresis loops (longitudinal and transverse) simulated for the equilateral hexagon prepared from nickel. While for the $0^{\circ}$ orientation, the transverse magnetization $M_{T}$ shows a broad range in which it does not saturate, clearly broader than

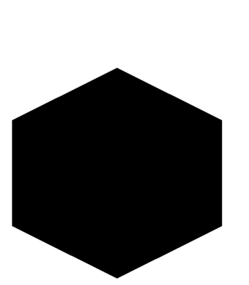

(a)

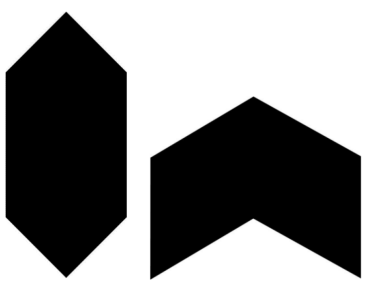

(b)

(c)
Fig. 1. Shapes used for the simulations in this paper (not drawn to scale): (a) equilateral, (b) rectangular, (c) concave.
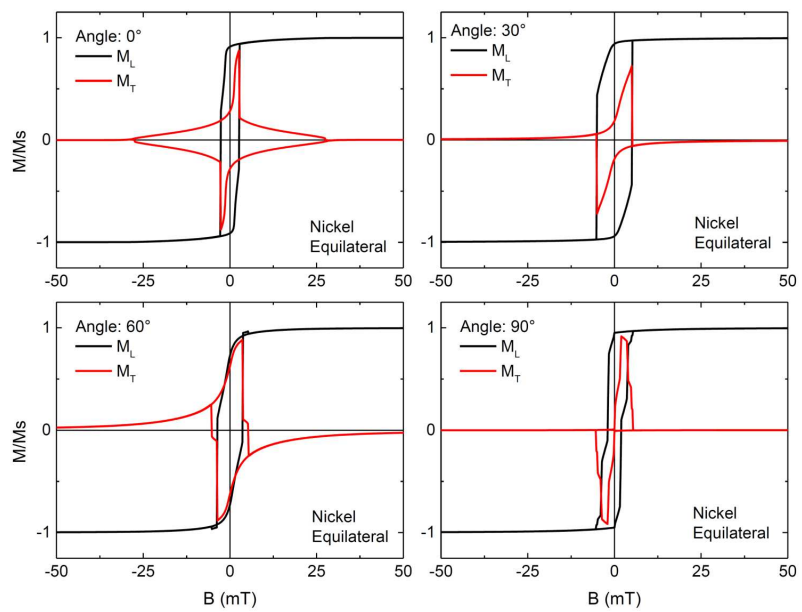

Fig. 2. Longitudinal $\left(M_{L}\right)$ and transversal hysteresis loops $\left(M_{T}\right)$, simulated for the equilateral hexagon prepared from nickel.

visible in $M_{L}$, both values are similar for the other angles under examination. For $60^{\circ}$ and $90^{\circ}$ angles, steps are visible along the slope of the hysteresis loop which will be examined further below.

Changing the material from nickel to iron, Fig. 3 shows broader hysteresis loops and more steps along the slopes of the hysteresis loops. In all angles besides $30^{\circ}$, comparing transversal and longitudinal hysteresis loops shows that magnetization reversal is not finished when the longitudinal loop seems to be closed, i.e. the external magnetic field necessary for saturation is much larger than the coercive fields.

This is also the case for the equilateral hexagon prepared from cobalt, except for the $90^{\circ}$ orientation (Fig. 4). Here, the hysteresis loops become even broader, as could be expected due to Co having the largest anisotropy constant. In addition, the angles of $60^{\circ}$ and $90^{\circ}$ show even more steps than the same shape prepared from Fe.

Next, Fig. 5 depicts exemplary hysteresis loops, simulated for the rectangular shape prepared from nickel. While the $0^{\circ}$ orientation shows a longitudinal hysteresis curve which is nearly closed and could be misinterpreted as superparamagnetic, the transversal curve shows clearly a coherent rotation of the whole magnetization, 

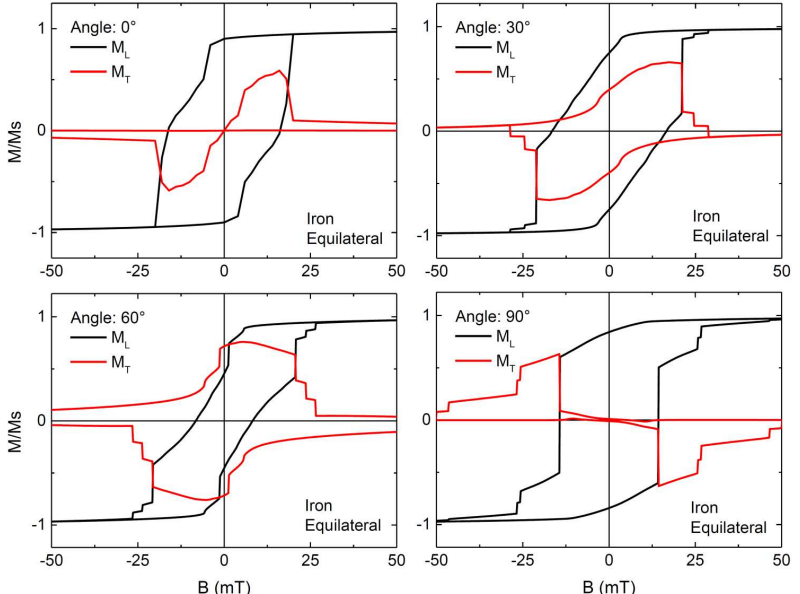

Fig. 3. Longitudinal and transverse hysteresis loops, simulated for the equilateral hexagon prepared from iron.
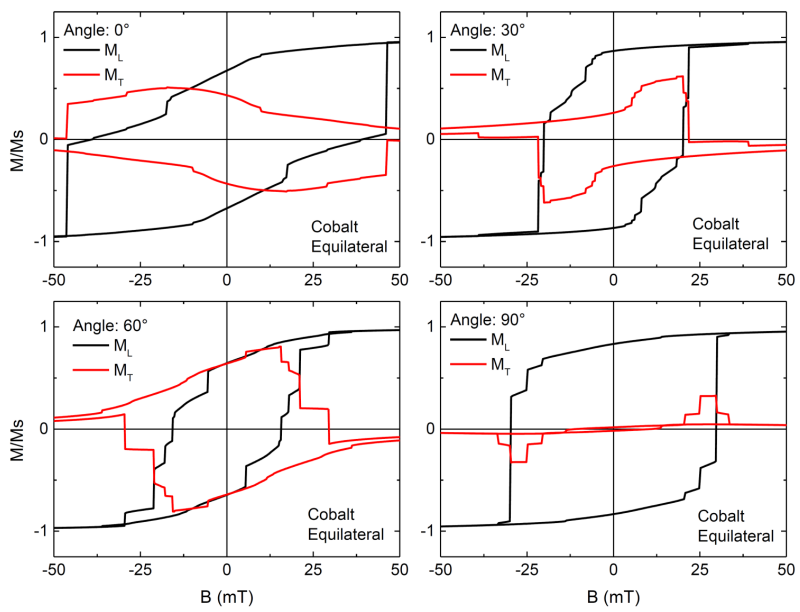

Fig. 4. Longitudinal and transversal hysteresis loops, simulated for the equilateral hexagon prepared from cobalt.

with the maximum values reaching 1 , i.e. around vanishing external magnetic field, the magnetization is completely oriented perpendicular to the field. The same effect is visible for $30^{\circ}$, while the maximum magnetization is slightly smaller for the $60^{\circ}$ orientation and nearly vanishes for $90^{\circ}$, indicating that here domain wall processes play a significant role.

For iron, the rectangular hexagon again shows several steps along the hysteresis loops, for $0^{\circ}$ again combined with the effect that the longitudinal loop seems to be closed at smaller external magnetic fields than the transversal one (Fig. 6).

For cobalt, again even more steps are visible in all angular orientations, as presented in Fig. 7. It should be mentioned that some of them are too small to be of technological relevance since for such nanomagnets, it is always necessary to take into account small deviations of
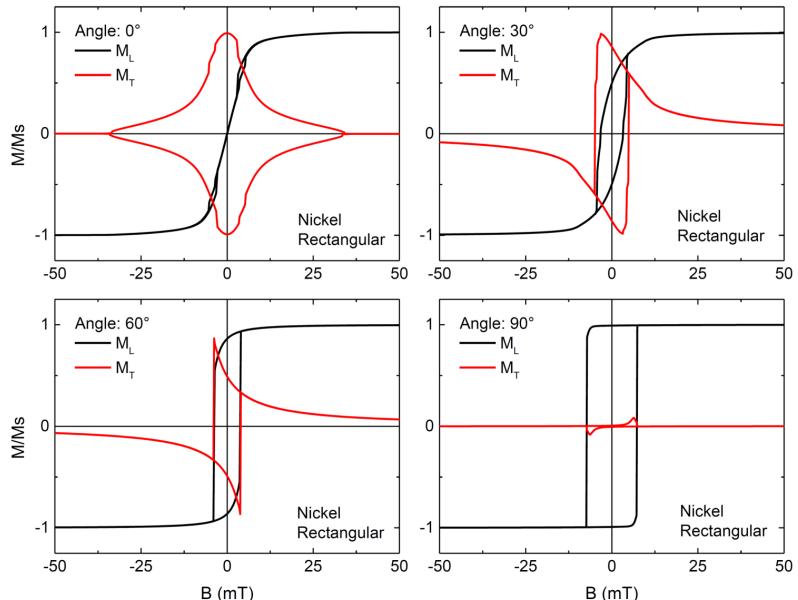

Fig. 5. Longitudinal and transversal hysteresis loops, simulated for the rectangular hexagon prepared from nickel.
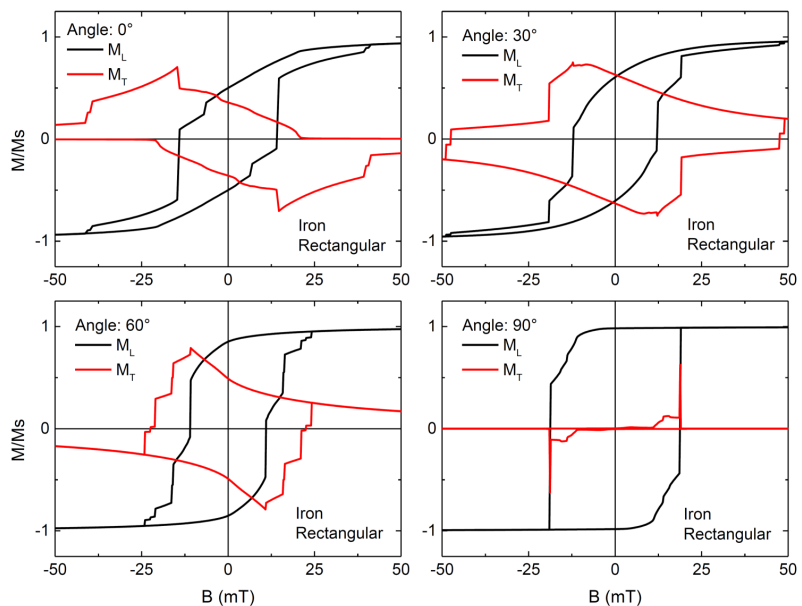

Fig. 6. Longitudinal and transversal hysteresis loops, simulated for the rectangular hexagon prepared from iron.

the shape due to the lithography process. This indicates that stopping and reversing the external magnetic field at a certain step is only possible if this step is broad enough; else the different states at remanence may be inseparable. In addition, it must be tested whether these states are not only stable at remanence, but can also be distinguished in a measurement.

For the concave hexagon, the simulations of nickel again show clear broad transverse peaks and corresponding steps in the hysteresis loops for all angles but $0^{\circ}$ (Fig. 8), indicating the possibility to use these steps for quaternary data storage applications.

For iron, Fig. 9 shows unusual longitudinal and transversal hysteresis loops, but not with significantly increased numbers of steps, indicating that this shape may be not favorable in comparison with both other nanoparticle shapes. 

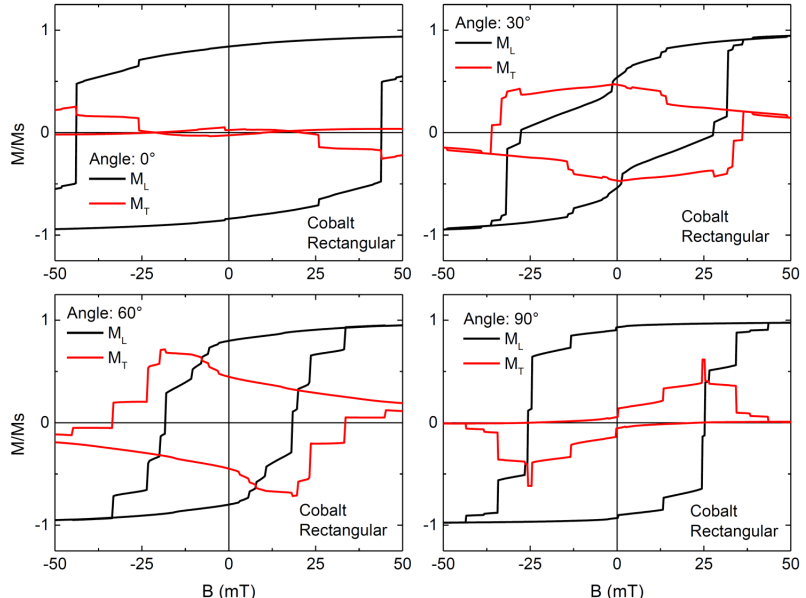

Fig. 7. Longitudinal and transversal hysteresis loops, simulated for the rectangular hexagon prepared from cobalt.
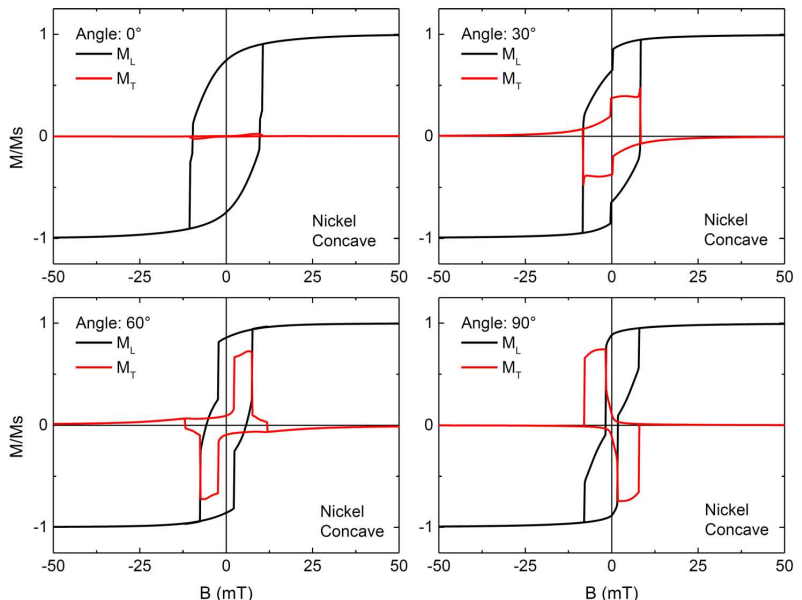

Fig. 8. Longitudinal and transversal hysteresis loops, simulated for the concave hexagon prepared from nickel.

Finally, the concave hexagon prepared from Co (Fig. 10) depicts several steps in the longitudinal and the transverse hysteresis loops, offering several potentially stable states at remanence especially for the angles of $30^{\circ}$ and $60^{\circ}$.

To visualize the differences between the three materials, Fig. 11 shows exemplary magnetization reversal processes of the rectangular hexagon under a field orientation of $90^{\circ}$. For nickel, only a small deviation from the orientation of the external magnetic field ("vertical") is visible before magnetization is reversed.

For iron, this effect is much stronger pronounced. Here, the final state before complete magnetization reversal shows a strong meander, comparable to the horseshoe state which can occur in open square frames and similar nanoshapes.

Cobalt, on the other hand, behaves differently. Even in the saturated case (left image), it is clearly visible that
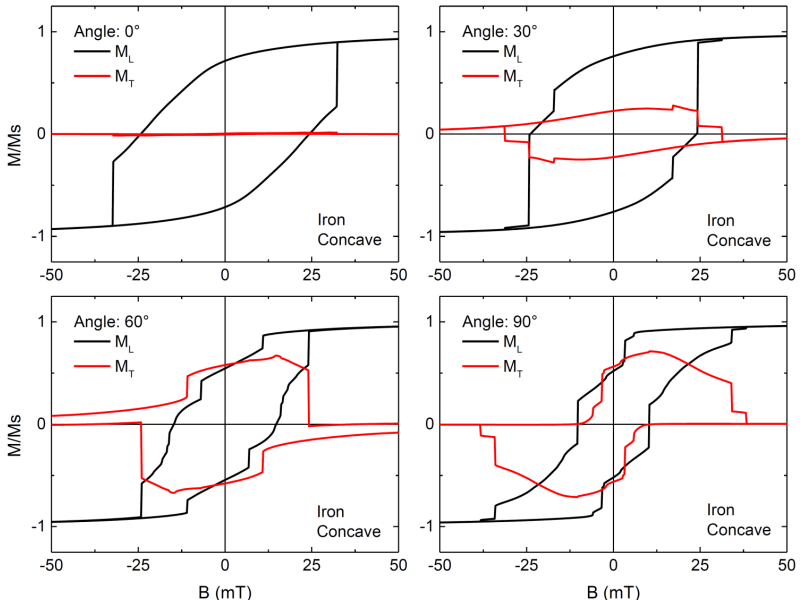

Fig. 9. Longitudinal and transversal hysteresis loops, simulated for the concave hexagon prepared from iron.
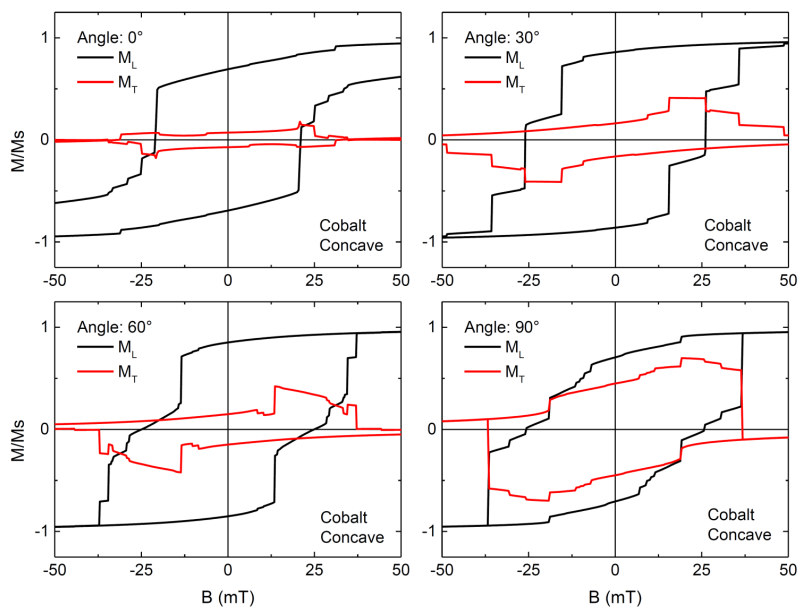

Fig. 10. Longitudinal and transversal hysteresis loops, simulated for the concave hexagon prepared from cobalt.

the magnetization orientation differs in several small areas, arbitrarily distributed along the nanomagnet. With reduced external magnetic field, some of these regions — which are not clearly separated by domain walls become more dominant and integrate other, sometimes smaller areas. Before the main step of the magnetization reversal occurs (from image 3 to image 4), there are also meander structures visible, but less structured than for the Fe nanomagnet. It should be mentioned that here still small areas are visible with the magnetization orientation differing from the surrounding material. Magnetization reversal thus does not occur at once, as for iron or nickel, but in several steps, as visible in the 4th image in which most of the magnetization is switched, but several areas are still oriented against the external magnetic field which is now oriented from top to bottom. In the last image depicted here, magnetization reversal is still not completed; at the bottom corner a small area still has to switch. 
$\mathrm{H}_{\mathrm{ext}}$

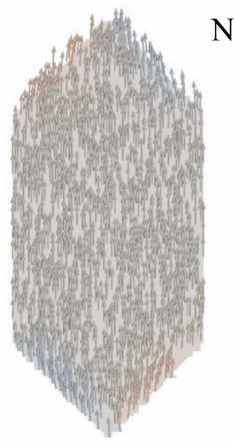

$\mathrm{Ni}$
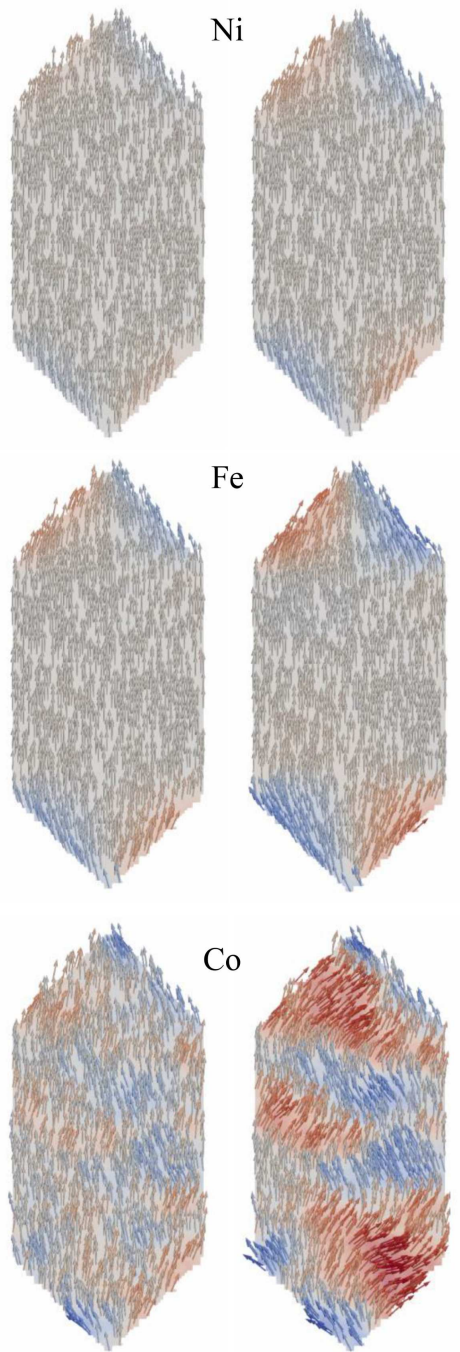

Co

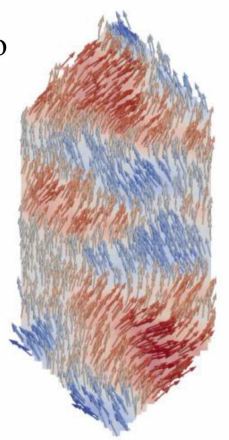

$\mathrm{Fe}$
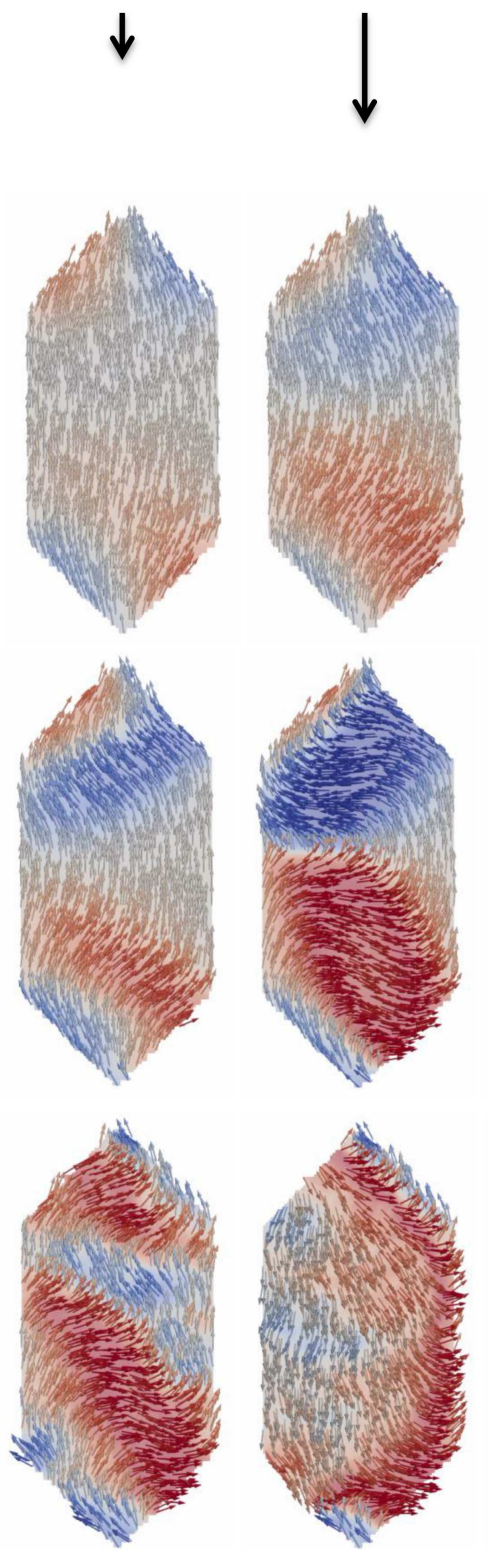

$\downarrow$

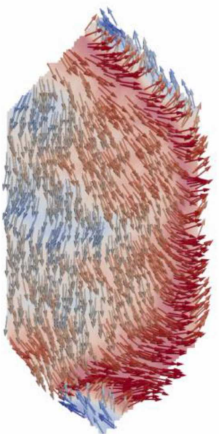

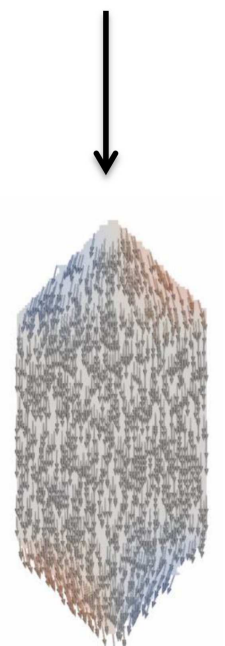
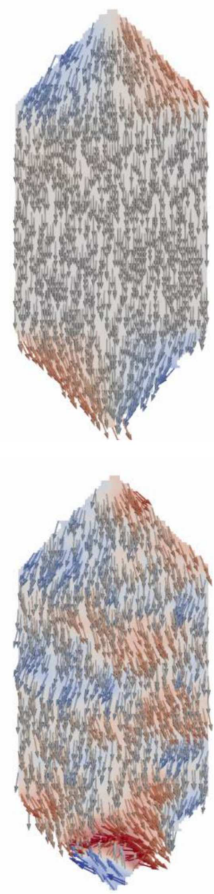

Fig. 11. Snapshots of key points of the magnetization reversal processes for $\mathrm{Ni}, \mathrm{Fe}$, and Co in the rectangular hexagon for the external magnetic field oriented along $90^{\circ}$. Magnetization orientation sweeping from "up" to "down" (see inset at the top). Color code: red - magnetization pointing to the right, blue - magnetization pointing to the left. The full videos are available as supplementary material.

These images indicate that Co is not the ideal material for nanostructures in the dimensions examined here. The most interesting material for these dimensions seems to be iron, while for thinner structures, cobalt can be expected to show sufficiently high magnetocrystalline anisotropy for an adequate interaction with the shape anisotropy, as it is the case here for iron.

An overview of the aforementioned discrepancies between coercive fields $H_{C}$ and fields necessary to reach saturation $H_{\text {Sat }}$ is given in Fig. 12 for the three materials and the three shapes under examination. In several cases, the $0^{\circ}$ orientation shows a large deviation between $H_{C}$ and $H_{\text {Sat }}$. In some cases, an additional maximum of this difference is reached at angles slightly larger than $60^{\circ}$, apparently correlated with the shape anisotropy of these hexagonal nanostructures. For Co, especially the saturation fields depict strong fluctuations for similar angles, indicating that for this material the magnetocrystalline anisotropy is dominating over the shape anisotropy, as already expected from Fig. 11. This underlines that for sputtered Co nanostructures of similar dimensions as examined here, the varying axes of the magnetocrystalline anisotropy in the grains of the single particles will reduce the reproducibility of their magnetic properties and thus the possible applications in data storage. 

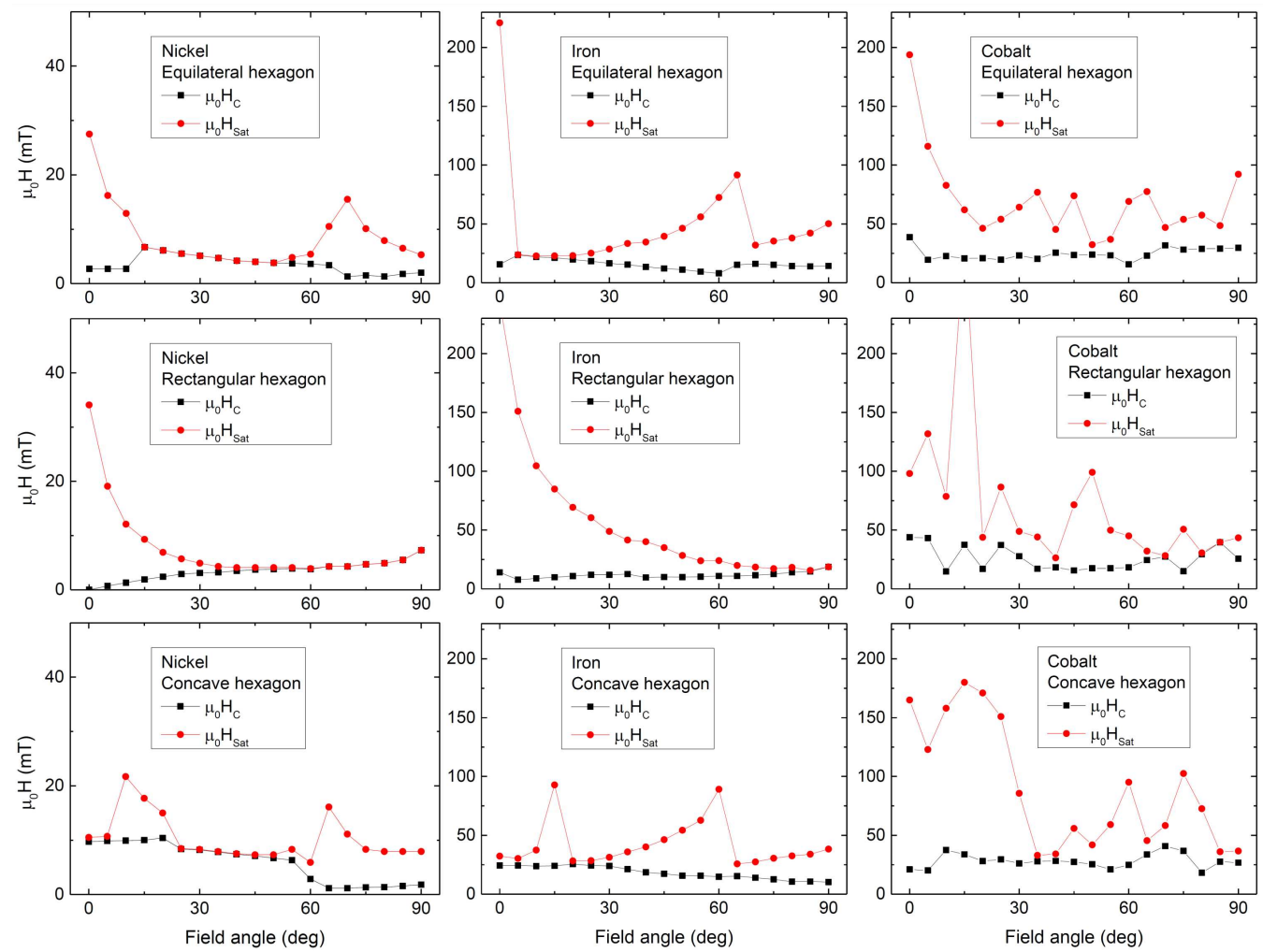

Fig. 12. Coercive fields $\mu_{0} H_{C}$ and saturation fields $\mu_{0} H_{\text {Sat }}$, simulated for the three different shapes prepared from the three different materials.
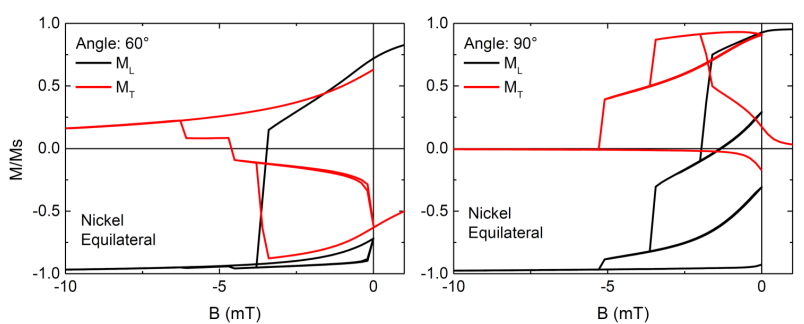

Fig. 13. Longitudinal and transverse minor loops, simulated for the equilateral hexagon prepared from nickel.

On the other hand, the nickel and iron samples show angular ranges with stable, predictable coercive and saturation fields. Especially the angles in which both values do not differ too strongly — which would necessitate too large writing fields - are of interest for a magnetic memory, provided that the steps in the hysteresis loops are correlated with stable intermediate states.

Tests of the stability of these possible intermediate states were performed by starting the simulation at positive saturation, stopping the field sweep in the first step, sweeping the field back to zero, going further to the second step, back to zero, etc. Situations depicted in Figs. 2-10 without any steps in the longitudinal or transversal loops were correspondingly not investigated.
Figure 13 shows the results of the stability tests of the equilateral nickel hexagon. For both angles, not all steps are stable; partly the longitudinal or transverse loops "jump" back into a former state before the magnetic field vanishes.

More interesting results can be found for the same shape, prepared from iron (Fig. 14). Here, e.g., the $90^{\circ}$ orientation shows five states at remanence, i.e. four states from magnetization reversal from positive to negative saturation and the first state of the way back from negative to positive saturation.

In spite of the abovementioned expected problems, the equilateral hexagon was also investigated for Co (Fig. 15). Here it becomes clearly visible that the states are not stable, but seem to jump back to former states at arbitrary external magnetic fields.

For the rectangular hexagon, nickel did not reveal any steps in the hysteresis loops. For iron (Fig. 16), $0^{\circ}$ and $30^{\circ}$ orientations show more than one stable state at remanence per field sweep direction, while a few closed loops indicate that not all steps belong to stable intermediate states.

For cobalt, more stable states are visible (Fig. 17); however, comparing this graph with the actually identical situation depicted in Fig. 7 shows clear deviations, underlining that this material is not reliable enough for data storage applications using nanoparticles of the relative large dimensions examined here. 

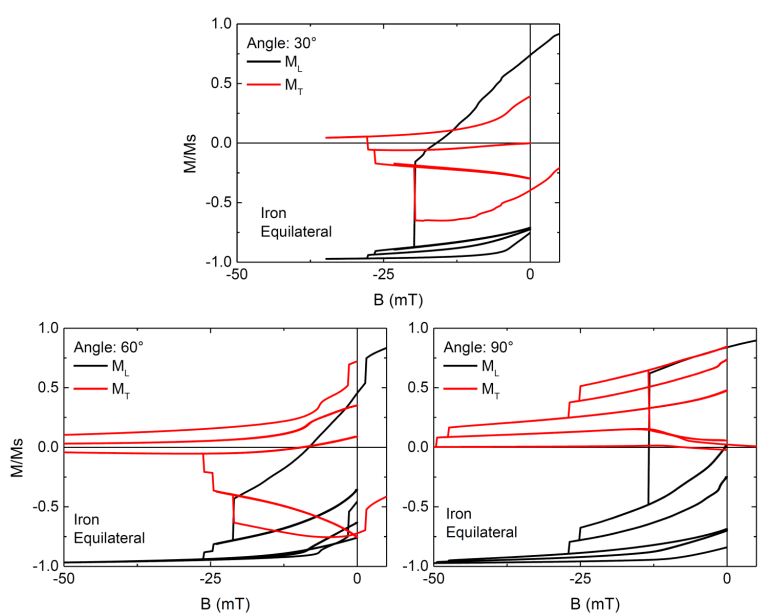

Fig. 14. Longitudinal and transversal minor loops, simulated for the equilateral hexagon prepared from iron.
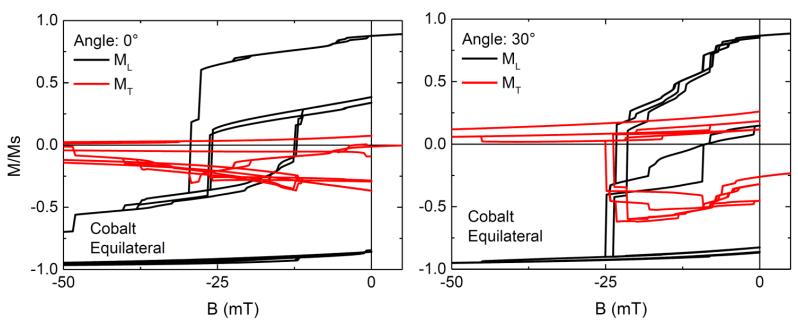

Fig. 15. Longitudinal and transversal minor loops, simulated for the equilateral hexagon prepared from cobalt.
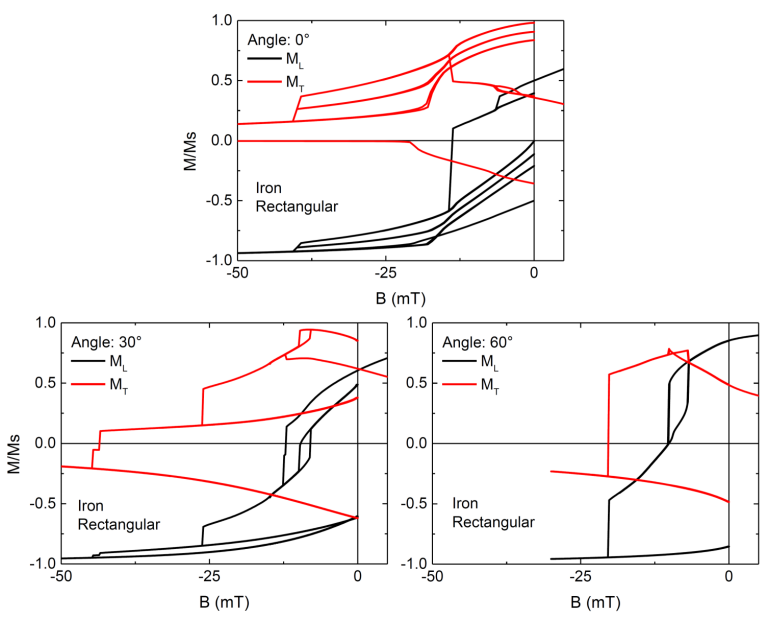

Fig. 16. Longitudinal and transversal minor loops, simulated for the rectangular hexagon prepared from iron.

For the concave hexagon, thus only iron was examined with respect to the stability of the intermediated states (Fig. 18). Again, not all steps are correlated with stable intermediate states, but nevertheless several such states are visible.

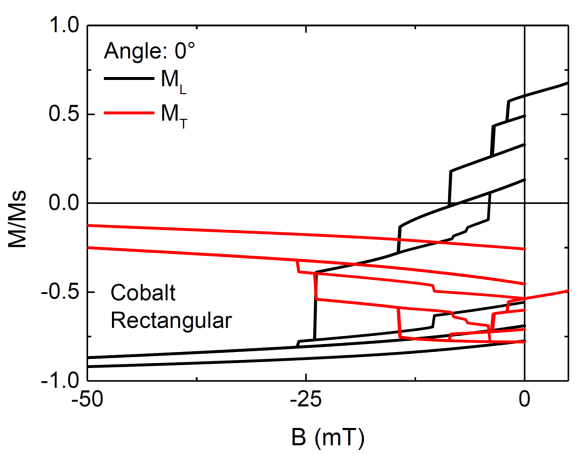

Fig. 17. Longitudinal and transversal minor loops, simulated for the rectangular hexagon prepared from cobalt.
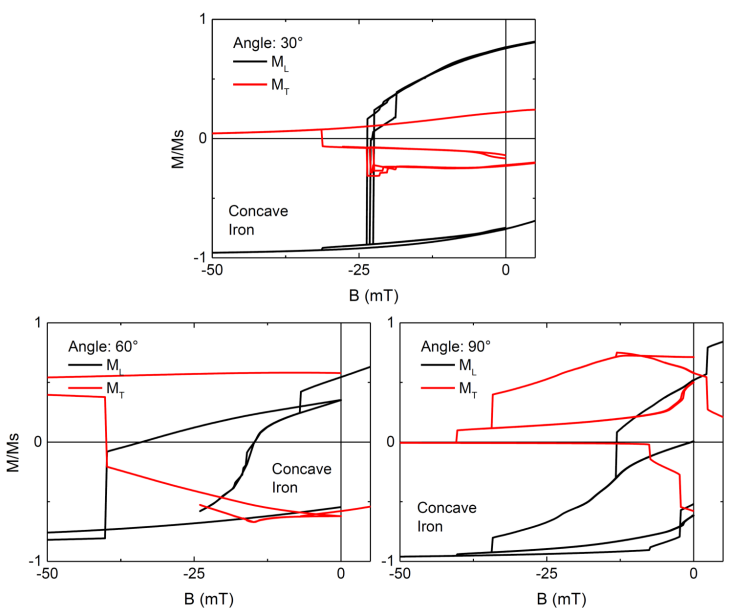

Fig. 18. Longitudinal and transversal minor loops, simulated for the concave hexagon prepared from iron.

Finally, the question arises whether it is possible to distinguish between the stable intermediated states, making them really usable technologically. As an example, Fig. 19 depicts the intermediate states simulated for the equilateral iron nanoparticle. Here, the longitudinal curve shows three very similar magnetizations, while the transverse curve includes a value near zero which cannot be used if both field sweep directions are planned to be utilized to reach stable intermediated states. On the other hand, it is known that measuring the magneto-optic Kerr effect (MOKE) can be used to detect the magnetization along all possible in-plane orientations [23]. Figure 19 thus depicts a series of graphs in which the detection direction rotates from $0^{\circ}\left(M_{L}\right)$ and $90^{\circ}\left(M_{T}\right)$, respectively, to $90^{\circ}\left(M_{T}\right)$ and $180^{\circ}\left(-M_{L}\right)$, giving an overview of all possible measurements.

To interpret these graphs, it should be mentioned that the single lines visible on the right side of the $y$-axis show the magnetization for the magnetic field decreasing from saturation, i.e. belong to the full hysteresis loop, and have their counterpart in one of the lines on the left side which is also part of the full hysteresis loop, while the additional stable states at remanence are reached by minor 

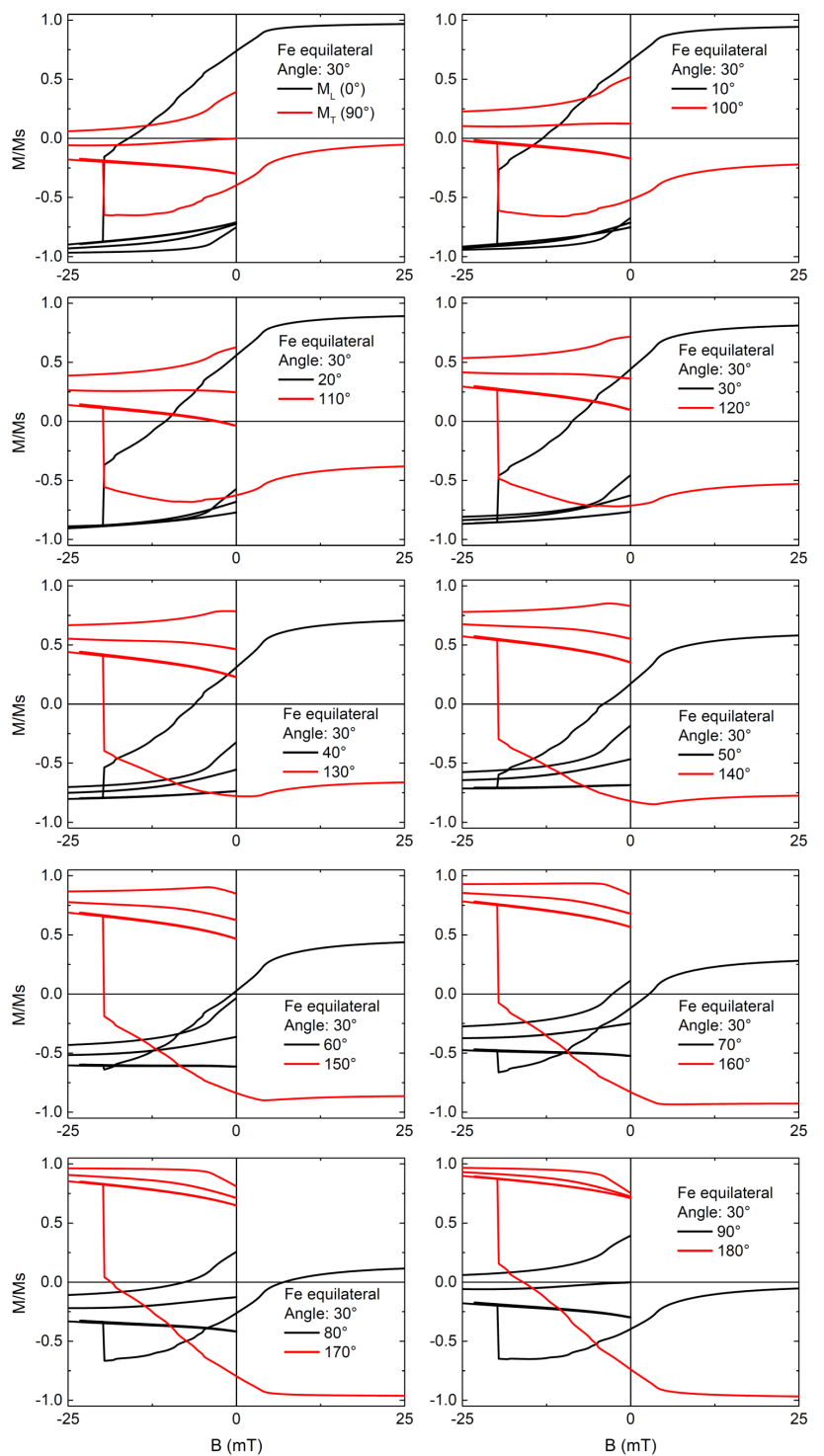

Fig. 19. Minor loops, simulated for the equilateral hexagon prepared from iron under an angle of $30^{\circ}$ to the external magnetic field, depicted for a spectrum of possible measurement orientations, measured with respect to the orientations of $M_{L}$ and $M_{T}$, respectively.

loops. These stable states are ideally detectable if they are well distributed over the whole $y$-axis. For $0^{\circ}$, e.g., three of the lines are very close at remanence, making this angle not well-suited.

For $90^{\circ}$, another problem occurs - here, one of the minor loops ends very near the coordinate origin. By adding all minor loops with opposite sign - starting from negative saturation - there will thus be two minor loops ending near the coordinate origin which will not be easily detectable.

Combining these two possible problems which can make the definite identification of a state at remanence complicated or impossible leads to the requirement to have all states (including those not depicted here, stemming from minor loops starting at negative saturation) as broadly distributed as possible along the $y$-axis.

Following this series of measurement orientations, it is clearly visible that a measurement orientation of $120^{\circ}$ $130^{\circ}$ is well suited to detect the different stable states at remanence unambiguously. The observation that a relatively large angular range around $120^{\circ}-150^{\circ}$ is generally suited for this purpose underlines that even in case of expectable shape deviations in real samples, there will be possibilities to use such systems for storing more than only 1 bit. Generally, most cases will offer similar opportunities to distinguish between the stable intermediate states and thus to use such nanomagnets as quaternary or higher-order data storage systems.

\section{Conclusion}

Different hexagonal nanomagnets prepared from nickel, iron, and cobalt were investigated by micromagnetic simulations. While the nickel nanoparticles showed simple magnetization reversal processes, often based on coherent rotation of the magnetization, the strong magnetocrystalline anisotropy of cobalt prohibited reliable magnetization reversal processes for sputtered samples, i.e. nanomagnets with arbitrary orientations of the single magnetic cells. For the sample dimensions investigated here in the range of some hundred nanometers, iron showed most promising numbers of stable intermediate states which could be distinguished from each other unambiguously. This makes especially the equilateral iron hexagon an interesting nanoparticle for the development of quaternary or higher order data storage devices.

\section{Acknowledgments}

This work was supported by Volkswagen Foundation grant "Adaptive Computing with Electrospun Nanofiber Networks" no. 93679.

\section{References}

[1] H.J. Li, Q. Wu, M. Yue, Y. Peng, Y.Q. Li, J.M. Liang, D.J. Wang, J.X. Zhang, J. Magn. Magn. Mater. 481, 104 (2019).

[2] M. Tadic, S. Kralj, Y. Lalatonne, L. Motte, Appl. Surf. Sci. 476, 641 (2019).

[3] C.C. Faulkner, M.D. Cooke, D.A. Allwood, D. Petit, D. Atkinson, R.P. Cowburn, J. Appl. Phys. 95, 6717 (2004).

[4] P. Kern, C. Döpke, T. Blachowicz, P. Steblinski, A. Ehrmann, J. Magn. Magn. Mater. 484, 37 (2019).

[5] T. Blachowicz, A. Ehrmann, J. Appl. Phys. 124, 152112 (2018).

[6] C. Garg, S.-H. Yang, T. Phung, A. Pushp, S.S.P. Parkin, Sci. Adv. 3, e1602804 (2017).

[7] R. Moreno, V.L. Carvalho-Santos, A.P. Espejo, D. Laroze, O. Chubykalo-Fesenko, D. Altbir, Phys. Rev. B 96, 184401 (2017). 
[8] J. Grollier, P. Boullenc, V. Cros, A. Hamzic, A. Vaurès, A. Fert, G. Faini, Appl. Phys. Lett. 83 509 (2003).

[9] N. Vernier, D.A. Allwood, D. Atkinson, M.D. Cooke, R.P. Cowburn, Europhys. Lett. 65, 526 (2004).

[10] S.-H. Yang, K.-S. Ryu, S.S.P. Parkin, Nat. Nanotechnol. 10, 221 (2015).

[11] S. Munjal, N. Khare, Appl. Phys. Lett. 113, 243501 (2018).

[12] T. Blachowicz, A. Ehrmann, J. Appl. Phys. 110 073911 (2011).

[13] T. Blachowicz, A. Ehrmann, J. Magn. Magn. Mater. 331, 21 (2013)

[14] R. Hertel, J. Appl. Phys. 90, 5752 (2001).

[15] A. Ehrmann, T. Blachowicz, Hyperfine Interact. 239 8 (2018).

[16] D.A. Bizyaev, A.A. Bukharaev, A.P. Chuklanov, N.I. Nurgazizov, Phys. Solid State 60, 2194 (2018).
[17] F.J. Castano, C.A. Ross, A. Eilez, J. Phys. D Appl. Phys. 36, 2031 (2003).

[18] A. Ehrmann, T. Blachowicz, AIP Adv. 5, 097109 (2015).

[19] M.J. Donahue, D.G. Porter, OOMMF User's Guide, Version 1.0 Interagency Report NISTIR 6376 National Institute of Standards and Technology, Gaithersburg 1999.

[20] S. Michea, J. Briones, J.L. Palma, R. Lavín, J. Escrig, R. Rodríguez-Suárez, J.C. Denardin, J. Phys. D Appl. Phys. 47, 335001 (2014).

[21] E.F. Kneller, R. Hawig, IEEE Trans. Magn. 27, 3588 (1991).

[22] I.Z. Rahman, A. Boboc, K.M. Razeeb, M.A. Rahman, J. Magn. Magn. Mater. 290-291, 246 (2005).

[23] A. Tillmanns, S. Oertker, B. Beschoten, G. Güntherodt, C. Leighton, I.K. Schuller, J. Nogués, Appl. Phys. Lett. 89, 202512 (2006). 